Original paper

\title{
Dose calculation accuracy in proximity of a pacemaker: A multicenter study with threecommercial treatment planning systems
}

\author{
Anna Delana ${ }^{a, *}$, Agnese Barbareschi $^{\mathrm{b}}$, Rita Consorti ${ }^{\mathrm{c}}$, Maria Daniela Falco ${ }^{\mathrm{d}}$ \\ ${ }^{\text {a }}$ Department of Medical Physics, S.Chiara Hospital, APSS Trento, Italy \\ ${ }^{\mathrm{b}}$ Department of Medical Physics, ASST Spedali Civili di Brescia, Brescia, Italy \\ ${ }^{c}$ Department of Medical Physics, San Filippo Neri Hospital ASL Roma 1, Rome, Italy \\ d Department of Radiation Oncology, "G. D'Annunzio" University, "SS. Annunziata” Hospital, Chieti, Italy
}

\section{A R T I C L E I N F O}

\section{Keywords:}

Out-of-field dose

Pacemaker

Radiotherapy

Treatment planning

System

Dose calculation accuracy

\begin{abstract}
A B S T R A C T
This study compares Treatment Planning System (TPS) out of field dose calculation on a pacemaker (PMK) during external beam radiotherapy treatment. We consider four TPSs (Elekta-Monaco, Oncentra- Masterplan and two Philips-Pinnacle3) commissioned for two linacs (Elekta Sinergy and Varian Clinac) delivering two test beams (a highly modulated one and a square field) and two clinical breast plans. To calculate and measure dose to a PMK we built a Real Water3 phantom with a PMK embedded in it. Measures are performed with thermoluminescent dosimeters and Mosfet dosimeters. We evaluate differences between TPS calculated values for the dose to the PMK (both point dose and dose-volume histogram parameters) when the PMK is positioned in the first $10 \mathrm{~cm}$ outside the radiation fields. TPS calculation accuracy is evaluated comparing such values with measures. Differences in TPS calculations are on average $3.5 \mathrm{cGy} \mathrm{Gy}^{-1}$ for the modulated beam, and always lower than 2 $\mathrm{cGy} \mathrm{Gy}^{-1}$ for the square beam. TPS dose calculation depends mostly on the TPS algorithm and model rather than the linac commissioned. TPSs considered show different degrees of calculation accuracy. In the first $4 \mathrm{~cm}$ to the field edge three out of four TPSs are in good agreement with measurements in the square beam, but only one keeps the agreement in the modulated beam: the others show over and underestimations up to $+20 \%-40 \%$. The same accuracy is found considering a homogeneous phantom. Our results confirm what reported in previous studies and highlight the impact of TPS commissioning.
\end{abstract}

\section{Introduction}

The use of implantable cardiovascular electronic devices (CIED) such as pacemakers (PMK) and defibrillators (ICD) has increased over the last decades. An increasing number of patients with CIEDs is therefore referred to radiotherapy centers as both conditions, the "dependency on a CIED" and "cancer diagnosis", are more frequent with older age and therefore overall frequency increases in an aging society [1].

When exposed to high energy radiation, CIEDs may encounter various malfunctions at doses exceeding $2 \mathrm{~Gy}$, such as reset to back-up mode, deprogramming or signal interference even if the devices are not directly exposed to the radiation field $[2,3]$. Neutrons produced by the interaction of photon beams at energy $>10 \mathrm{MV}$ with heavy metals in the LINAC head are believed to be the main cause of CIED defects $[3,4]$; therefore, many guidelines consider a maximum energy of $6 \mathrm{MV}$ optimal for treating patients with CIEDs [5-10].
Clinical guidelines [5,8-10] provide guidance for patient management and identify risk classes considering both clinical and dosimetric aspects of the radiotherapy (RT) treatment plan. Low risk is associated with patients that are not pacemaker-dependent and with CIED maximum doses $<2$ Gy. Medium risk is assigned to patients not pacemaker-dependent with CIED maximum doses between $2 \mathrm{~Gy}$ and 10 Gy and patients pacemaker-dependent receiving a CIED maximum dose less than 2 Gy. Patients who receive a CIED maximum dose of $10 \mathrm{~Gy}$ or above are classified as high risk. Thus, the dose delivered to the CIED plays an important role in the categorization of these patients and medical physicists must carefully estimate it. The aforementioned guidelines suggest that, in order to diminish the dose received by the CIED, it should be kept outside the primary radiation field [2,5,8-10].

The estimation of CIED dose should be performed with the use of "in vivo" dosimetry and/or a treatment planning system (TPS) [7]. As reported in AAPM TG 158 [11], TPSs may show large calculation errors at

\footnotetext{
* Corresponding author.

E-mail address: anna.delana@apss.tn.it (A. Delana).
} 
distances $>3 \mathrm{~cm}$ from the field edge and/or below the $5 \%$ isodose line, $\mathrm{i}$. e. in the region where a CIED is typically located relative to the target volume $[6,7]$. In the first $10 \mathrm{~cm}$ from the field edge the components of the out-of-field dose are, in order of relevance, patient scatter, collimator scatter and head leakage. Particularly the last two components are likely not being modelled properly by the TPS [12]. As stated in AAPM 203 [8] another source of error arising in the TPS's calculation is the presence of high-density objects and CIEDs usually have metal components and a metal case.

TPS dose calculation accuracy in the out-of-field region has already been investigated [12-16] but specific TPSs dose error estimation in proximity of a high-density object and in particular relative to CIED structures have not yet been performed [17]. As medical physicists usually consider dose volume histograms (DVH) to estimate the maximum and mean dose received by CIEDs, DVH dose calculation errors must be evaluated too. Most reports concerning out of-field-dose [12-16] consider only one TPS commissioned for one linac (mostly Varian Clinac), therefore there is a need for a study that compares several TPSs and considers Elekta linacs as well.

The aim of this multicenter study is to compare out-of-field dose calculation results in proximity of a CIED for a variety of TPSs currently in clinical use as well as the calculation of CIED DVHs (CIED maximum dose and mean dose). The second part of this work then evaluates CIED dose calculation accuracy when compared to TLD- and MOSFET-based phantom measurements.

\section{Materials and methods}

\subsection{Phantom, imaging, and treatment planning}

To reproduce the position of the CIED, in this case a pacemaker (PMK) in the human body and to measure the dose to the PMK, we used an in-house built phantom. The phantom consists of a PMK embedded in a PMMA slab with $2 \mathrm{~cm}$ of PTW Real Water 3 (RW3) positioned above (CIEDs are usually placed between 1 and $3 \mathrm{~cm}$ beneath the skin [8]) and $10 \mathrm{~cm}$ of RW3 below (Fig. 1). The PMK was covered with wax to avoid air gaps and to host three holes for the dosimeters to accurately reproduce their positioning during dose verification at the linac (Fig. 1 (a)). These holes were placed above the PMK: one approximately at its proximal end (P1), one in the middle (P2), one at the distal end (P3). CT images of this phantom were acquired with a Philips Brilliance BigBore (120 kVp, $250 \mathrm{mAs} /$ slice, $3 \mathrm{~mm}$ slice thickness).Center C1 delineated the structures PMK, CT artifacts and the positions P1, P2 and P3. CT datasets and structures were then sent to all participating centers in DICOM format. Density correction was performed only for CT artifacts. For the PMK each TPS used its CT to ED calibration curve and calculation grid used in clinical practice (for details see Table 1).

Three commercial TPSs commissioned for two linac models were included in the study. Table 1 summarizes TPS, dose calculation algorithms, maximum value of CT-ED calibration curve, calculation grid resolution used and linacs for which they were commissioned. TPS P $3_{\mathrm{E}}$ is currently used only for 3D conformal planning, while the other TPSs are also used for Intensity Modulated RT (IMRT) and volumetric modulated arc therapy (VMAT) plans.

\subsection{Test plans}

To compare out of field dose values calculated by different TPSs for non-modulated and highly-modulated beams, we considered two simple test plans that could be easily created on each TPS. The non-modulated beam was a $10 \times 10 \mathrm{~cm}^{2}$ square beam. The highly-modulated beam was a $10 \times 10 \mathrm{~cm}^{2}$ modulated field as a series of 10 rectangular strips in the linac's $\mathrm{AB}$ direction of $10 \mathrm{~cm} \times 1 \mathrm{~cm}$ each and a beam modulation (BM) of 0.9 as defined by Du et al [18]. Each plan had a prescription dose of 2 Gy at the isocenter, at SSD $95 \mathrm{~cm}$ and $6 \mathrm{MV}$ energy. Dose calculation is performed by shifting the beam isocenter in the linac's TG direction, in order to have the PMK proximal edge from $1 \mathrm{~cm}$ to $10 \mathrm{~cm}$ from the field edge (see Fig. 1). Center C1 also analyzed a clinical case of a left side breast cancer patient simulating two more plans: a 3D conformal plan with field in field technique, BM of 0.29 (with TPS $\mathrm{MC}_{\mathrm{E}}$ and $\mathrm{P} 3_{\mathrm{E}}$ ) and an IMRT plan with two dMLC beams and BM of 0.5 (with TPS MC $\mathrm{E}_{\mathrm{E}}$ ). The plans were calculated and optimized on a patient CT dataset, prescribing $50 \mathrm{~Gy}$ in 25 fractions, and then transferred and recalculated on the PMK phantom with the beams collapsed at gantry fixed at $0^{\circ}$.

\subsection{Phantom irradiation}

Dose measurements were performed with Lithium fluoride thermoluminescent dosimeters (Harshaw TLD-100 LiF, square rods with dimension of $6 \times 1 \times 1 \mathrm{~mm}^{3}$ ) at center $\mathrm{C} 1$ and Thomson Nielsen's mobile MOSFET Dose Verification System (TN-RD-70-W) with MOSFET dosimeters (TN-502RD, nominal sensitivity $1 \mathrm{mV} / \mathrm{cGy}$ ) at center C3. These detectors have been shown to be accurate as out-of-field dosimeters operated at $6 \mathrm{MV}$ [20-22]. Detectors were inserted at each hole P1, $\mathrm{P} 2$ and $\mathrm{P} 3$, and measurements were repeated shifting the phantom in the linac's TG direction to measure the out of filed dose at increasing distance from the field edge $(1 \mathrm{~cm}, 3 \mathrm{~cm}, 5 \mathrm{~cm}$ and $10 \mathrm{~cm})$.

\subsubsection{Detector calibration}

For each measuring session, detector calibration was performed in RW3 slabs with a $10 \times 10 \mathrm{~cm}^{2}$ square $6 \mathrm{MV}$ beam, prescribing $2 \mathrm{~Gy}$ at the isocenter.

Before each calibration, TLDs were properly prepared and background radiation was estimated with 3 unexposed dosimeters and subtracted from the exposed TLD reading. TLD batch homogeneity response was less than $3 \%$. TL detectors are energy-dependent [23,24] and additional correction factors should be used to reduce the over-response at low energy spectrum. However, at the distances and depth considered in this experimental setup, TLD sensitivity was previously estimated to be close to unity [25], therefore we did not apply further corrections.

MOSFET calibration was performed with the 3 detectors used and each measurement was repeated 5 times. Before each measurement session, background radiation was estimated and taken into account by the measuring system. MOSFET reproducibility depends on dose levels and their age, reproducibility at dose levels of interest for this study ranges from $4 \%$ (at $10 \mathrm{cGy}$ ) to $15 \%$ (in the range $2-4 \mathrm{cGy}$ ) [21].

\subsubsection{Detector uncertainty estimation}

To achieve the highest possible accuracy for the measured dose, the signal was quantified as the average of repeated measurements (5 TLDs at each hole, MOSFET measures are repeated 3 times) and the uncertainty of the measurements was estimated as the standard deviation of the readings. The error due to the uncertainty of the positioning of the dosimeters was quantified with the formula:

error $\%=$ dosegradient $\left[\frac{\%}{\mathrm{~mm}}\right] *$ positioningerror $[\mathrm{mm}]$

where the dose gradient is estimated as the maximum difference between $\mathrm{D}(\mathrm{x}) / \mathrm{D}_{\mathrm{CAX}}$ measured in the first $3 \mathrm{~cm}$ from the beam edge and the positioning error is $3 \mathrm{~mm}$. The resulting dose uncertainty due to positioning error was less than $1 \%$. We also estimated the accuracy of the dosimeters at low doses by analyzing their reproducibility in the dose range of interest in our study.

Overall uncertainty, considering setup errors and low dose measure repeatability, was quantified as $9 \%$ for the TLD and ranging between $6 \%$ and $17 \%$ for doses between 2 and 12 cGy for MOSFET.

\section{Analyses performed}

All analyses were performed in terms of $\mathrm{D} / \mathrm{D}_{\mathrm{CAX}}(\mathrm{x})$, i.e. the dose at distance $\times$ to the beam edge normalized to the central axis dose D(CAX) 


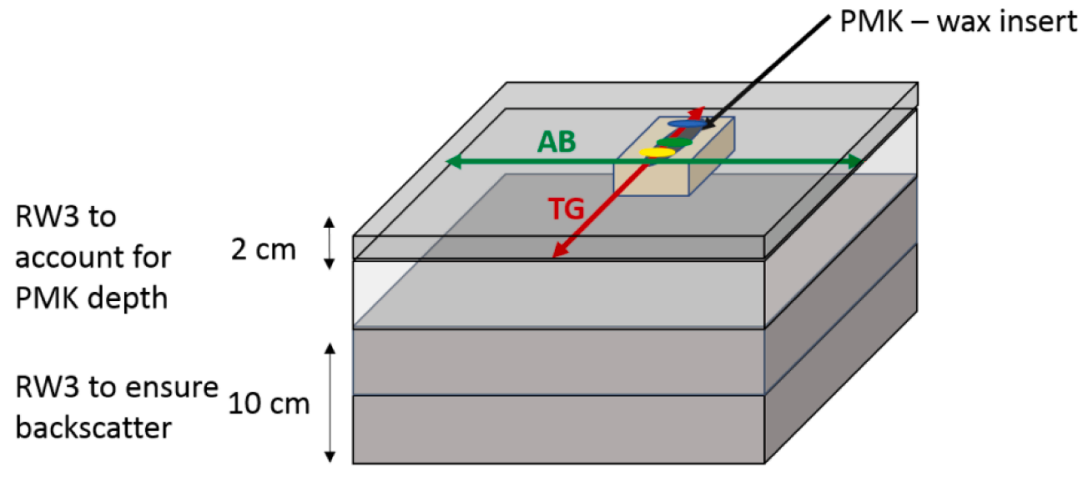

(a)

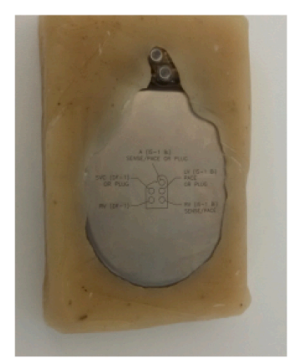

(b) Wax insert with PMK

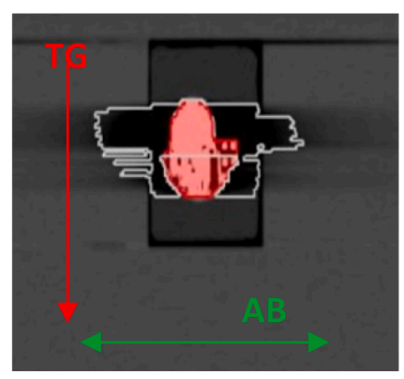

(c) Coronal view

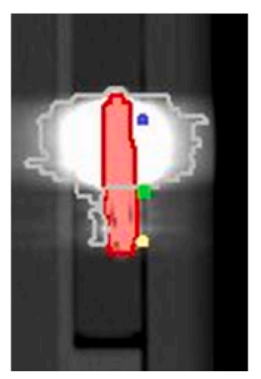

(d) Sagittal view

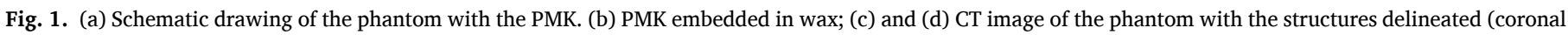

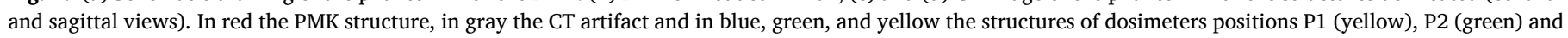
P3 (blue). (For interpretation of the references to colour in this figure legend, the reader is referred to the web version of this article.)

and expressed as $\%$ of $\mathrm{D}_{\mathrm{CAX}}$.

\subsection{TPS dose comparison}

\subsubsection{PMK dose analysis}

TPS dose comparison was performed in terms of dose to the PMK. We considered dose to P1, P2 and P3 as reported in Fig. 1 and, for PMK's DVH analysis, dose to $1 \mathrm{cc}$ of PMK structure $\left(\mathrm{D}_{1 \mathrm{cc}}\right.$ i.e. the maximum dose received by the PMK), and mean dose to the PMK $\left(D_{\text {mean }}\right)$. For each PMK position $x$ and each parameter considered (point dose or DVH parameter) we considered the maximum difference $\Delta D_{M A X}(x)$ between TPSs' calculated dose values

$\Delta D_{M A X}(x)=\max _{i, j \in\{1 a, 1 b, 2,3\}}\left|\left(D / D_{C A X}(x)\right)_{T i}-\left(D / D_{C A X}(x)\right)_{T j}\right|$

where the indexes $i$ and $j$ run over all TPSs combinations. These analyses were also performed by separating the analysis of the maximum absolute difference between $\mathrm{D} / \mathrm{D}_{\mathrm{CAX}}(\mathrm{x})$ calculated from TPSs with a CCC calculation algorithm for the Elekta linac $\left(\left|\mathrm{P}_{\mathrm{E}}-\mathrm{MP}_{\mathrm{E}}\right|\right)$, and the two Philips - Pinnacle3 TPSs commissioned for different linacs $\left(\left|\mathrm{P} 3_{\mathrm{E}}-\mathrm{P} 3_{\mathrm{V}}\right|\right)$. Analyses done for the test plans are performed also for the 3D-CRT clinical breast beams considering TPSs $\mathrm{MC}_{\mathrm{E}}$ and $\mathrm{P} 3_{\mathrm{E}}$.

\subsubsection{Comparison with homogeneous phantom}

We evaluated the impact of the PMK's electron density on dose calculation calculating D/D $\mathrm{D}_{\mathrm{CAX}}$ with each TPS on a homogeneous RW3 phantom at the same distances to the field edge. Then, we analyzed the difference in the $\mathrm{D} / \mathrm{D}_{\mathrm{CAX}}$ values estimated from each TPS on the two phantoms. As both $\mathrm{P} 3_{\mathrm{E}}$ and $\mathrm{P} 3_{\mathrm{V}}$ are Pinnacle3 TPSs, only $\mathrm{P} 3_{\mathrm{V}}$ was considered for this analysis.

\subsection{TPS dose accuracy}

\subsubsection{PMK dose analysis}

TPS accuracy was quantified as the ratio of calculated to measured values $\frac{D_{T P S}}{D_{\text {meas }}}$

$\frac{D_{T P S}}{D_{\text {meas }}}=\frac{\left(D / D_{C A X}\right)_{T P S}}{\left(D / D_{C A X}\right)_{\text {measure }}}$

To estimate DVH parameter calculation accuracy, we first estimated the Person's correlation coefficients between $\mathrm{D}_{1 \mathrm{cc}}$ and the calculated P1 dose and between $\mathrm{D}_{\text {mean }}$ and the mean of the calculated point doses. We evaluated TPSs DVH accuracy comparing measured dose at P1 with $\mathrm{D}_{1 \mathrm{cc}}$.

We also investigated if beam modulation affect TPS calculation accuracy.

\subsubsection{Comparison with homogeneous phantom}

We compared TPS out-of-field dose accuracy in presence of a PMK and in homogenous medium by quantifying $\frac{D_{T P S}}{D_{\text {meas }}}$ for point dose in the two phantoms, for each TPS and for the square and IMRT beams.

\section{Results}

\subsection{TPS dose calculation comparison}

\subsubsection{PMK dose analysis}

Fig. 2(a) shows the point dose calculated by the TPSs and Fig. 2(b) shows $\Delta D_{M A X},\left|\mathrm{P} 3_{\mathrm{E}}-\mathrm{MP} \mathrm{P}_{\mathrm{E}}\right|$ and $\left|\mathrm{P} 3_{\mathrm{E}}-\mathrm{P} 3_{\mathrm{V}}\right|$ as a function of the distance from the beam edge for the IMRT field. Discrepancies between all TPSs $\left(\Delta D_{\text {MAX }}\right)$ have a mean value of $3.5 \%(+/-0.7 \%, 1 \mathrm{SD})$ and are higher far 
Table 1

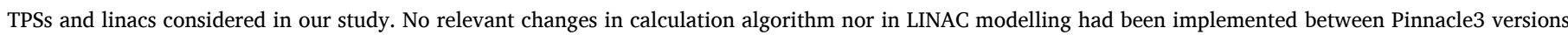
considered. XVMC: Voxel Monte Carlo [19]; CCC: Collapsed Cone Convolution.

\begin{tabular}{|c|c|c|c|c|c|c|}
\hline Centers & TPS & & Algorithm & Max CT to ED value & Calculation Grid & LINAC \\
\hline C1 & $\mathrm{MC}_{\mathrm{E}}$ & Elekta Monaco v.5.11 & XVMC & 3.8 & $2 \mathrm{~mm}$ & Elekta Synergy Agility \\
\hline $\mathrm{C} 1$ & $\mathrm{P} 3_{\mathrm{E}}$ & $\begin{array}{l}\text { Philips } \\
\text { Pinnacle3 v.9.8 }\end{array}$ & CCC & 4.6 & $2 \mathrm{~mm}$ & Elekta Synergy Agility \\
\hline $\mathrm{C} 2$ & $\mathrm{MP}_{\mathrm{E}}$ & $\begin{array}{l}\text { Elekta } \\
\text { Oncentra Masterplan v.4.5.3 }\end{array}$ & CCC & 2.8 & $2 \mathrm{~mm}$ & Elekta Synergy Agility \\
\hline C3 & $\mathrm{P} 3{ }_{\mathrm{V}}$ & $\begin{array}{l}\text { Philips } \\
\text { Pinnacle3 v.9.10 }\end{array}$ & $\mathrm{CCC}$ & $1.8^{* * *}$ & $3 \mathrm{~mm}^{* * *}$ & Varian Trilogy \\
\hline
\end{tabular}

** TPS P3V performed the calculations also with a $2 \mathrm{~mm}$ grid and CT to EC density curve of 4.6 like the other Philips Pinnacle TPS P $3_{\mathrm{E}}$.

from the beam edge; $\left|\mathrm{P} 3_{\mathrm{E}}-\mathrm{MP}_{\mathrm{E}}\right|$ and $\left|\mathrm{P} 3_{\mathrm{E}}-\mathrm{P} 3_{\mathrm{V}}\right|$ are always less than $2 \%$ and $\left|\mathrm{P} 3_{\mathrm{E}}-\mathrm{P} 3_{\mathrm{V}}\right|$ is always lower than $0.5 \%$. Higher $\triangle D_{M A X}$ values are related to TPS $\mathrm{MC}_{\mathrm{E}}$ (XVMC algorithm) with respect to the CCC TPSs: XVMC calculates a higher dose with respect to the other TPSs.

$\Delta D_{M A X}$ values for the square field are always less than $2 \%$ (not reported in a figure) and are higher closer to the beam axis. At distances greater than $4 \mathrm{~cm} \Delta D_{\text {MAX }}$ decreases to an almost constant value of $0.5 \%$.

In Fig. 3 PMK $\mathrm{D}_{1 \mathrm{cc}}$ are reported as a function of the PMK's distance from the beam edge; PMK $\mathrm{D}_{1 \mathrm{cc}}$ are normalized to the central axis dose and differences between TPSs values are not shown. In the square beam, $\mathrm{D}_{1 \mathrm{cc}}$ shows almost the same behavior for all TPSs. $\mathrm{D} / \mathrm{D}_{\mathrm{CAX}}$ differences are lower than $1.5 \%$ when considering all TPSs and lower than $0.5 \%$ when considering Pinnacle3 TPSs. Comparing TPSs commissioned to the same linac model in the IMRT field, $\mathrm{MC}_{\mathrm{E}}$ shows the same behavior as TPS $\mathrm{P} \mathrm{B}_{\mathrm{E}}$ up to $7 \mathrm{~cm}$ with a maximum difference of $0.7 \%$, and it calculates a higher dose than $\mathrm{MP}_{\mathrm{E}}$.

$D_{\text {mean }}$ has a similar behavior to $D_{1 c c}$ (graphs are not reported) but differences between TPSs are lower: for the square beam TPSs have discrepancies of less than $1 \%$ of $\mathrm{D}_{\mathrm{CAX}}$, and less than $3.5 \%$ of $\mathrm{D}_{\mathrm{CAX}}$ in the IMRT field. $\mathrm{D}_{1 \mathrm{cc}}$ for the two breast plans has the same behavior as the square test beam. Differences in the TPSs' calculated $\mathrm{D}_{1 \mathrm{cc}}$ parameter are greater closer to the field edge, with a maximum value of $1.7 \%$, while differences in the calculation of $D_{\text {mean }}$ are almost constant with a value of $0.2 \%$.

\subsubsection{Comparison with homogeneous phantom}

Fig. 4 shows the out-of-field $\mathrm{D} / \mathrm{D}_{\mathrm{CAX}}$ calculated in the two phantoms as a function of the beam edge distance. Differences in the $\mathrm{D} / \mathrm{D}_{\mathrm{CAX}}$ values calculated on the two phantoms are almost negligible for both fields and always less than $1.6 \%$.

\subsection{TPS dose calculation accuracy}

\subsubsection{PMK dose analysis}

We compared TPS- calculated D/ $\mathrm{D}_{\mathrm{CAX}}$ for positions $\mathrm{P} 1, \mathrm{P} 2$ and $\mathrm{P} 3$ with measurements as shown in Table 2. TPS $\mathrm{MC}_{\mathrm{E}}$ is in very good agreement with measurements for the square beam, whereas for the IMRT plan the agreement is worse. TPS $\mathrm{P} 3_{\mathrm{V}}$ has good agreement between measured and calculated $\mathrm{D} / \mathrm{D}_{\mathrm{CAX}}$ for both plans.

For the two breast plans (3D-CRT and $\mathrm{dMLC}$ ) TPS $\mathrm{MC}_{\mathrm{E}}$ calculates a slightly higher $\mathrm{D} / \mathrm{D}_{\mathrm{CAX}}$ than the measured value, while for TPS $\mathrm{P} 3_{\mathrm{E}}$ this is true only for $\mathrm{D} / \mathrm{D}_{\mathrm{CAX}}$ greater than $1.5 \%$.

Fig. 5 shows the average value of $\frac{D_{T P S}}{D_{\text {meas }}}$ calculated over all the POIs considered as a function of BM. To further compare our results with the study of Huang et al. [12], we plot them as a function of MU/Gy.

\subsubsection{DVH dose analysis}

Pearson's correlation coefficient between $\mathrm{P} 1$ dose and $\mathrm{D}_{1 \mathrm{cc}}$ ranges from 0.97 to 0.99 so we used P1 dose measurements to evaluate the accuracy of this DVH parameter. Pearson's correlation coefficient between the average value of P1, P2 and P3 doses and $\mathrm{D}_{\text {mean }}$ ranges from 0.83 to 0.99 , therefore they are not linearly correlated, and we evaluated calculation accuracy only for the calculated point dose and not for this DVH parameter.

Table 2 also shows the average ratio of $\frac{\mathrm{D}_{\mathrm{TPS}}}{\mathrm{D}_{\text {meas }}}$ values for $\mathrm{P} 1$ that we took as the reference dose for $\mathrm{D}_{1 \mathrm{cc}}$. Both fields have the largest discrepancy far from the field, with the PMK at $5 \mathrm{~cm}$ to the beam edge.
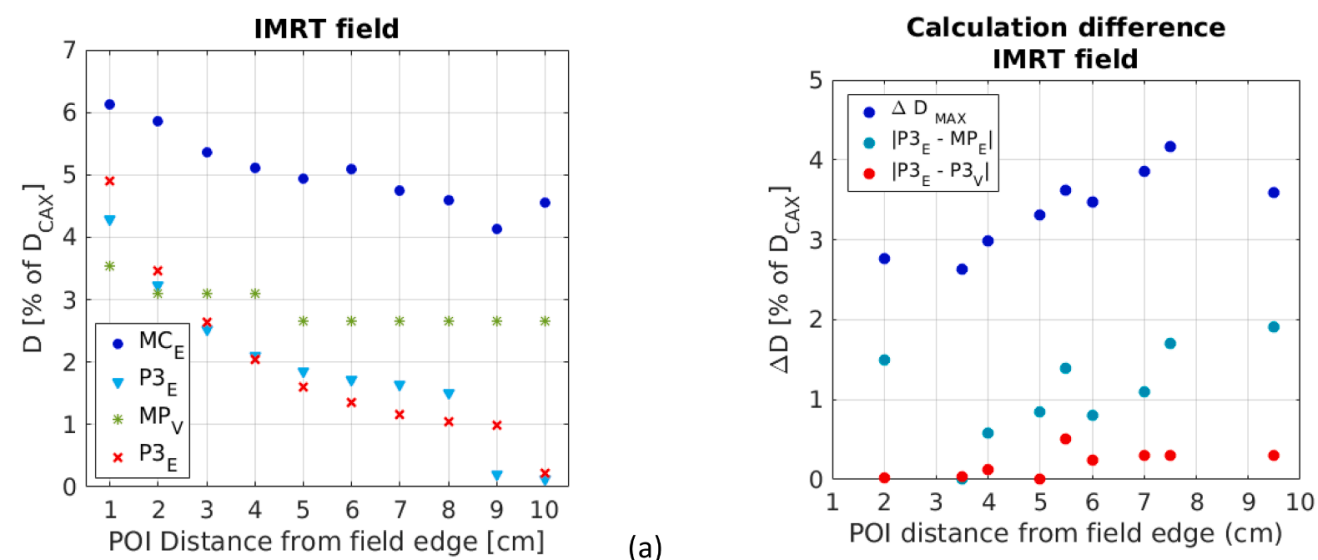

(b)

Fig. 2. (a) Point dose at different distances from the beam edge for the IMRT beam (b) Maximum difference between calculated isodose at all points for the IMRT plan. Blue dots represent the maximum discrepancy between calculated isodose of all TPSs $\left(\Delta \mathrm{D}_{\mathrm{MAX}}\right)$, light blue dots are the difference between TPSs with two CCC calculation algorithms commissioned for the Elekta linac $\left(\left|\mathrm{P} 3_{\mathrm{E}}-\mathrm{MP} \mathrm{P}_{\mathrm{E}}\right|\right)$, and red dots are the difference between Pinnacle3 TPSs commissioned for two different linacs $\left(\left|\mathrm{P} 3_{\mathrm{E}}-\mathrm{P} 3_{\mathrm{V}}\right|\right)$. (For interpretation of the references to colour in this figure legend, the reader is referred to the web version of this article.) 
D1Cc square beam

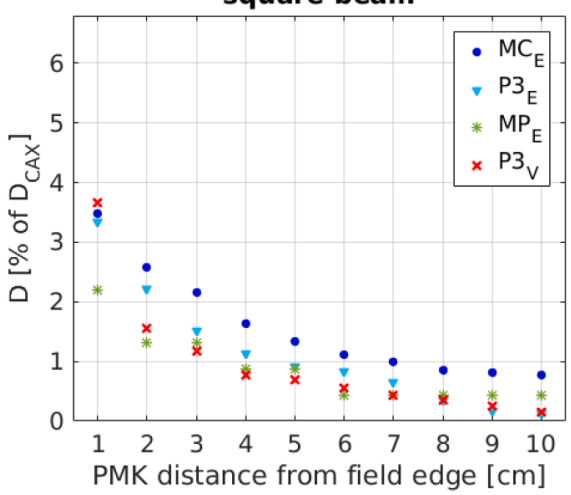

D1cc

IMRT beam

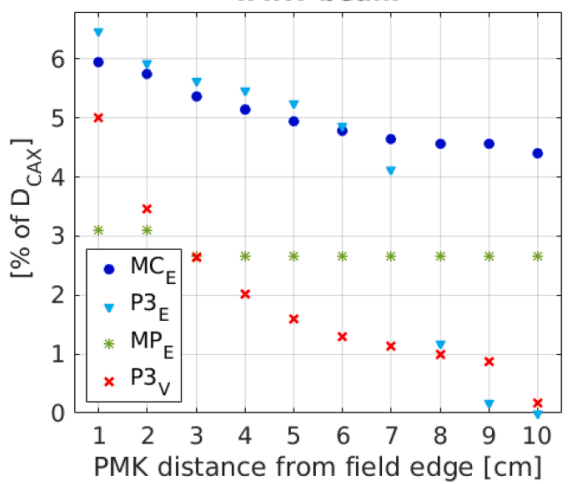

(b)

Fig. 3. $\mathrm{D}_{1 \mathrm{cc}}$ at various PMK distances from the field edge, as calculated by TPSs included in our study for the square (a) and the IMRT (b) $10 x 10 \mathrm{~cm}^{2}$ beam. Values of $\mathrm{D}_{\text {mean }}$ have the same behavior.

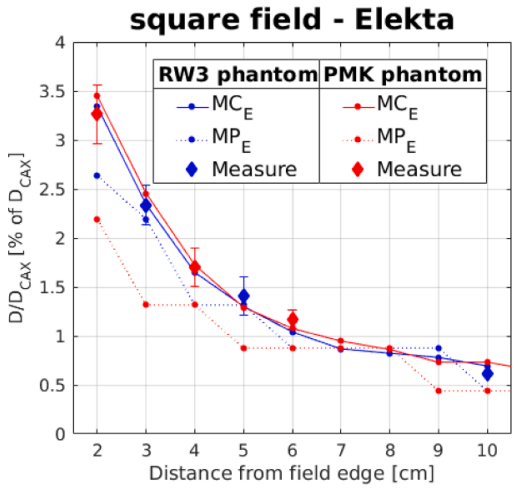

(a)

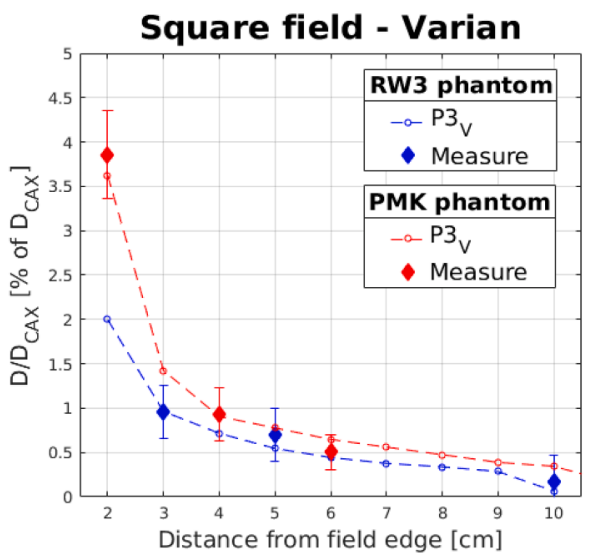

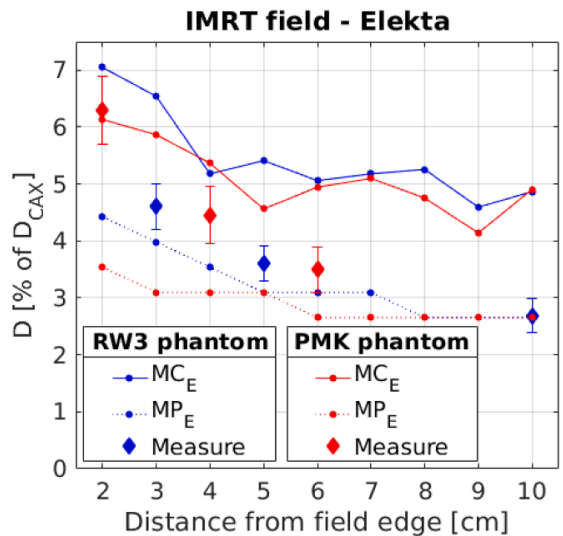

(b)

(c)

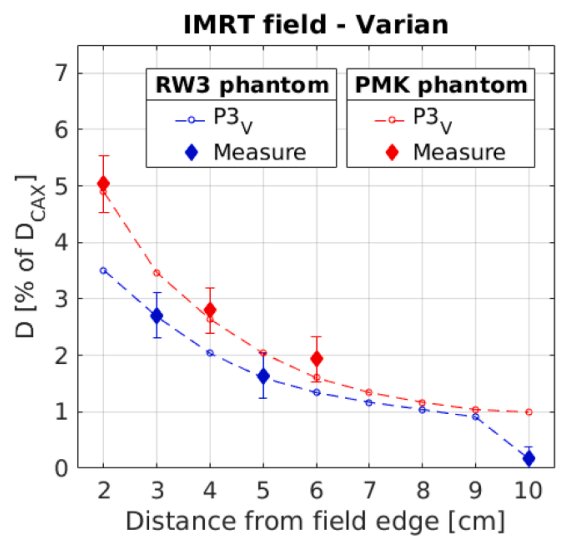

(d)

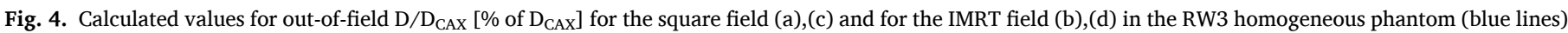

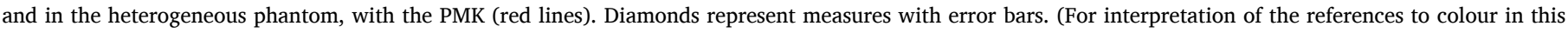
figure legend, the reader is referred to the web version of this article.)

\subsubsection{Comparison with homogeneous phantom}

Fig. 6 shows the average ratio of $\frac{D_{T P S}}{D_{\text {meas }}}$ on the two phantoms considered (homogeneous RW3 and with the PMK) for TPSs $\mathrm{MC}_{\mathrm{E}}, \mathrm{MP}_{\mathrm{E}}$ and $\mathrm{P} 3_{\mathrm{V}}$. In the PMK phantom only P1 is considered. Values on the two phantoms are within $1 \mathrm{SD}$ from each other (except $\mathrm{MP}_{\mathrm{E}}$ in the square field, 2SD) for both fields.

\section{Discussion}

Our study aimed to compare TPS dose calculation in the evaluation of dose to a PMK, considering both out of field dose points and DVH dose estimations. We investigated their accuracy by comparing calculated doses with measurements. We also evaluated the impact of the introduction of a high-density object such like a PMK on the accuracy of TPS calculation. 
Table 2

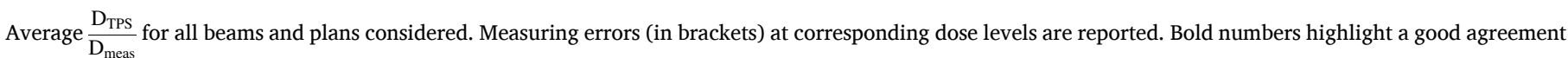
between calculated and measured values.

\begin{tabular}{|c|c|c|c|c|c|c|}
\hline & \multicolumn{3}{|l|}{ Square beam } & \multicolumn{3}{|l|}{ IMRT beam } \\
\hline & $1-4 \mathrm{~cm}$ & $4-10 \mathrm{~cm}$ & $\mathrm{P} 1$ & $1-4 \mathrm{~cm}$ & $4-10 \mathrm{~cm}$ & $\mathrm{P} 1$ \\
\hline $\mathrm{MC}_{\mathrm{E}}$ & $1.05( \pm 9 \%)$ & $0.99( \pm 9 \%)$ & $0.99( \pm 9 \%)$ & $1.18(+/ 9 \%)$ & $1.53( \pm 9 \%)$ & $1.23( \pm 9 \%)$ \\
\hline $\mathbf{P} 3_{\mathrm{E}}$ & $0.97( \pm 9 \%)$ & $0.65( \pm 9 \%)$ & $0.84( \pm 9 \%)$ & $0.62( \pm 9 \%)$ & $0.54( \pm 9 \%)$ & $0.59( \pm 9 \%)$ \\
\hline $\mathrm{MP}_{\mathrm{E}}$ & $0.64( \pm 9 \%)$ & $0.69( \pm 9 \%)$ & $0.72( \pm 9 \%)$ & $0.65( \pm 9 \%)$ & $0.83( \pm 9 \%)$ & $0.67( \pm 9 \%)$ \\
\hline $\mathbf{P 3} \mathbf{v}_{\mathrm{v}}$ & $\begin{array}{l}1.01( \pm 15 \%) \\
\text { 3D-CRT breast plan }\end{array}$ & $1.18( \pm 15 \%)$ & $1.07( \pm 17 \%)$ & $\begin{array}{l}0.97( \pm 12 \%) \\
\text { dMLC breast }\end{array}$ & $0.86( \pm 13 \%)$ & $0.93( \pm 13 \%)$ \\
\hline $\mathrm{MC}_{\mathrm{E}}$ & $1.10( \pm 9 \%)$ & $0.99( \pm 9 \%)$ & $1.05( \pm 9 \%)$ & $1.09( \pm 9 \%)$ & $0.85( \pm 9 \%)$ & $0.95( \pm 9 \%)$ \\
\hline $\mathbf{P} \mathbf{3}_{\mathrm{E}}$ & $1.12( \pm 9 \%)$ & $0.76( \pm 9 \%)$ & $0.93( \pm 9 \%)$ & - & - & - \\
\hline
\end{tabular}

In the calculation of the out-of-field dose, discrepancies between TPS's dose calculation are larger for the IMRT field than for the square field: the maximum difference found for the IMRT field is $4.7 \%$ whereas for the square field, differences are always lower than $1.5 \%$. When considering only CCC TPSs, differences are always lower than $2 \%$ regardless of the field considered. As shown in Fig. 2a. each TPS model has its own behavior. The two Pinnacle 3 TPSs $\left(\mathrm{P}_{\mathrm{E}}\right.$ and $\mathrm{P} 3_{\mathrm{V}}$ ) show a steep exponential decrease with distance to the $\mathrm{CAX}, \mathrm{MC}_{\mathrm{E}}$ shows a slower decrease in dose with distance and $\mathrm{MP}_{\mathrm{E}}$ returns a constant value of $2.7 \%$ after $4 \mathrm{~cm}$ to the field edge. Such different behaviors are caused by differences in the calculation of the patient and collimator scatter components by the TPSs. As reported previously $[12,26,27]$, type B TPSs underestimate these dose components when compared with Monte Carlo simulation. Collimator scatter strongly depends on the modulation of the field. The modulation of the IMRT field in our study is about 10 times greater than in the square field and therefore the biggest differences are found in the dose calculated for the IMRT field.

TPSs dose calculation accuracy was estimated by comparing dose calculated over the PMK structure with measurements, increasing the PMK's distance from the beam edge (results are reported in Table 2). TPSs $\mathrm{MC}_{\mathrm{E}}$ and $\mathrm{P} 3_{\mathrm{E}}$ show a better agreement with measurements in the square field and in the breast plans than in the IMRT field. TPS MPE always underestimates the measured dose and TPS $\mathrm{P} 3 \mathrm{~V}$ is always in good agreement with measurements. In the first $4 \mathrm{~cm}$ from the beam edge of the square beam (where the doses received by the PMK are of clinical relevance) all TPSs except $\mathrm{MP}_{\mathrm{E}}$ are in good agreement with measured data. At distances greater than $4 \mathrm{~cm}$ from the beam edge only TPSs $\mathrm{MC}_{\mathrm{E}}$ and $\mathrm{P} 3 \mathrm{~V}$ dose calculations are within our measurement error for the lowmodulated beams (square beam and breast plan). TPSs with CCC (apart from $\mathrm{P}_{\mathrm{V}}$ ) mostly underestimate the measurements, while the TPS with the XVMC dose calculation algorithm $\left(\mathrm{MC}_{\mathrm{E}}\right)$ mostly overestimates measurements. Deviations between calculated and measured dose values are almost always within acceptability criteria reported in IAEA TRS430 for the out-of-field dose region $[28,29]$. Our results with CCC TPSs are in accordance with previous studies that reported a TPS's

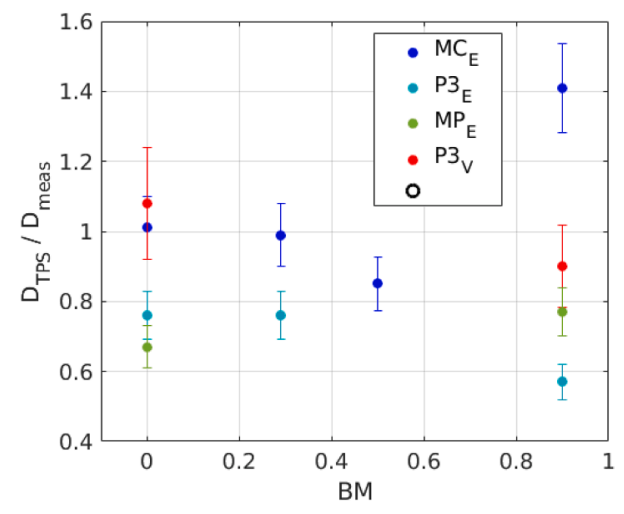

underestimation in the out-of-field dose region by up to $50 \%$ $[11-13,15,16,30]$. No studies have been performed with the XVMC calculation algorithm yet. Joosten compared a Monte Carlo model (BEAMnrc) with TLD measurements, and found differences in the range of $[-3 \%,+23 \%]$ in the first $10 \mathrm{~cm}$ from the field's edge, confirming our results of TPS's dose overestimation [27].

The two Pinnacle3 TPSs show different behaviors: $\mathrm{P} 3 \mathrm{~V}$ is always in good agreement with measured data, while $\mathrm{P} 3_{\mathrm{E}}$ always underestimates the measurements. We investigated if this difference in TPS accuracy was related to calculation grid size or maximum CT-ED value. TPS P $3 \mathrm{~V}$ recalculated dose distributions with calculation $2 \mathrm{~mm}$ grid size and with maximum CT-ED value equal to 4.6 , as TPS P3E: POI doses change at maximum of $0.04 \%$ of $\mathrm{D}_{\mathrm{CAX}}$ and $\mathrm{DVH}$ parameters $\mathrm{D}_{1 \mathrm{cc}}$ and $\mathrm{D}_{\text {mean }}$ differ at maximum of $0.3 \%$ of $D_{\mathrm{CAX}}$. Therefore the impact of the grid size and maximum CT-ED value on the TPS accuracy estimation is less than $1 \%$.

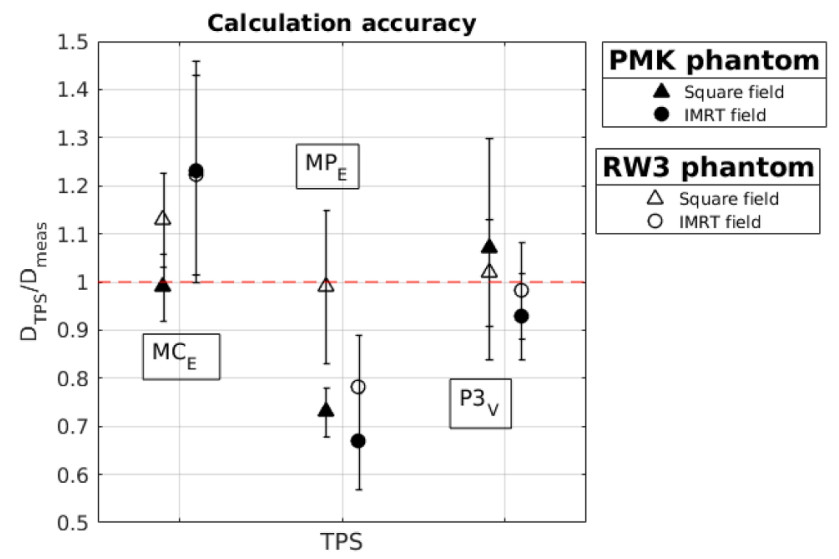

Fig. 6. Average $\frac{D_{\text {TPS }}}{D_{\operatorname{meas}}}$ values for the two test fields and the two phantoms. Error bars represent $1 \mathrm{SD}$ of the data.

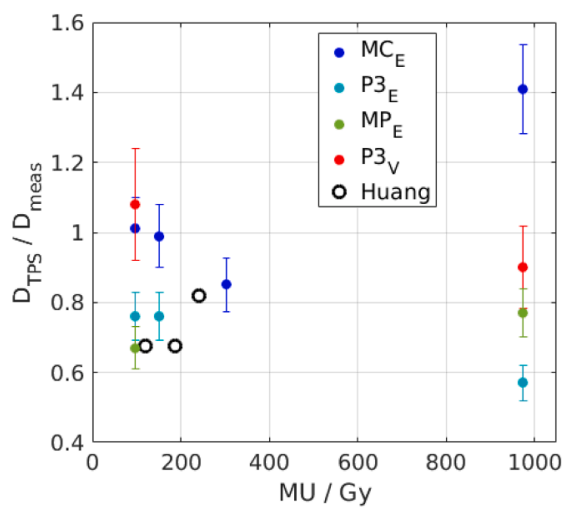

(b)

Fig. 5. TPS calculation accuracy as a function of beam modulation (a) and MU/Gy (b). Error bars represent 1SD of the data. 
Another aspect that we analyzed, is the accuracy of linac modeling for the two TPSs, during commissioning process: we quantified it by calculating the mean error between the measurements and calculated out-of-field doses. This error is 10 times lower for center C3 when compared to $\mathrm{C} 1$. The better performance of TPS $\mathrm{P} 3_{\mathrm{V}}$ could be related to a finer tuning of this TPS in the out-of-field region, during commissioning.

The fact that the TPS $\mathrm{MC}_{\mathrm{E}}$ - Monaco with XVMC calculation algorithm - shows a worse accuracy in the out-of-field dose calculation than TPS P $3_{V}$ - Pinnacle 3 with CCC calculation algorithm- can be explained by the fact that Pinnacle3 TPSs are tunable by the user, while this is not possible in Monaco. However, $\mathrm{MC}_{\mathrm{E}}$ dose calculation is a conservative estimate of the dose and such discrepancies with measured dose would not lead to clinically relevant issues. TPS MP $\mathrm{E}$ is not tunable by the user either, and shows an underestimation of the measured dose of an average $35 \%$.

Huang [12] in his study also considered plan modulation as a possible source of TPS's inaccuracy in out-of-field calculation but found no difference between plans with different modulation. Our results partially disagree with his results, as we find poorer calculation accuracy in the highly modulated beam and a good agreement with measurements for the low modulated fields for TPS $\mathrm{MC}_{\mathrm{E}}$ (XVMC calculation algorithm). TPSs with a CCC algorithm are in accordance with Huang's results (as reported in Fig. 5b). We can therefore suggest that a dependency of TPS accuracy on plan modulation may be related to the calculation algorithm.

Following AAPM 203 guidelines [8], medical physicists usually consider DVH parameters to estimate the dose received by the CIED. We therefore studied how different TPSs' calculation affects DVH dose estimation and the accuracy of DVH dose parameters $D_{1 c c}$ and $D_{\text {mean }}$. We first evaluated the clinical impact of differences in the calculated $\mathrm{D}_{1 \mathrm{cc}}$ and $D_{\text {mean }}$ (reported in Fig. 3) for a 25 -fraction treatment ( $2 \mathrm{~Gy} /$ fraction at the isocenter). For the square field $\mathrm{D}_{1 \mathrm{cc}}$ and $\mathrm{D}_{\text {mean }}$ differences are on average 48 cGy and 34 cGy, while for the IMRT field these differences can exceed $200 \mathrm{cGy}$ at all distances to the beam edge. Even between TPSs with the CCC algorithm commissioned for the same linac model $\left(\mathrm{P} 3_{\mathrm{E}}\right.$ and $\left.\mathrm{MP}_{\mathrm{E}}\right)$, differences are on average $150 \mathrm{cGy}$ for the IMRT field and $22 \mathrm{cGy}$ for the square field. This variability in DVH dose estimations could impact the patients risk class definition and therefore individual patients' management, especially for highly modulated plans.

We also studied the accuracy of DVH parameter $\mathrm{D}_{1 \mathrm{cc}}$ by analyzing the accuracy of POI P1 dose. When the PMK is positioned at $1 \mathrm{~cm}$ to the beam edge the calculated $\mathrm{P} 1$ dose values are consistent with measured dose for all TPSs except $\mathrm{MP}_{\mathrm{E}}$, for the square plan, and only for $\mathrm{MC}_{\mathrm{E}}$ and P 3 for the IMRT plan. The farther the PMK is positioned from the field edge, the worse agreement is found between $\mathrm{D}_{\text {TPS }}$ and $\mathrm{D}_{\text {MEAS. }}$. As shown in table 2 , TPS P $3 \mathrm{~V}$ is always within the dosimeter's uncertainties while TPS $\mathrm{MC}_{\mathrm{E}}$ is in good agreement with measured values for the low modulated beams and overestimates the measurements by an average value of $20 \%$ for the IMRT beam. This means that $D_{1 \text { cc }}$ is accurately estimated by TPSs $\mathrm{P}_{\mathrm{V}}$ and conservatively estimated by TPS MC $\mathrm{M}_{\mathrm{E}}$. Other TPSs underestimate the dose with respect to the measurements with an average value of $20 \%$ for the square field and $40 \%$ for IMRT. These underestimations, even if within tolerances of IAEA TRS430 for the outof-field dose region [28], must be considered when $\mathrm{D}_{1 \text { cc }}$ calculation is used for patient risk class assessment.

Tests performed in this study focused on two simple beams, which represent two extreme cases of plan's modulation and one clinical case (left breast treatment). As the highest source of uncertainty in the out-offield region arises from head scatter modeling [12,27], our IMRT beam (modulation of 0.9) might represent an extreme situation with respect to most clinical cases. Moreover, we decided to perform our tests at $2 \mathrm{~cm}$ depth, as to be within typical CIED positions inside the body and to mitigate the dosimetric effect of positioning errors that could arise in the build-up region. We therefore may assume that dosimetric uncertainties of PMK dose in clinical plans should stay within our results.

By comparing TPSs' calculation in presence of a PMK and in homogeneous medium, differences in the calculated $\mathrm{D} / \mathrm{D}_{\mathrm{CAX}}$ are almost negligible for all TPSs: for both fields the difference is always less than $1.6 \%$ of $\mathrm{D}_{\mathrm{CAX}}$, even though TPSs have different CT-ED calibration curves. For all TPSs considered and for both test beams, the agreement between the measured and calculated dose on the PMK phantom does not differ from that on the homogeneous RW3 phantom. $\frac{D_{T P S}}{D_{\text {meas }}}$ estimated on the RW3 homogeneous phantom are within 1SD from that estimated on the heterogeneous phantom, except for $\mathrm{MP}_{\mathrm{E}}$ for which the agreement is within 2SD (see Fig. 6). We can therefore assume that the presence of a high-density object in the out of field region does not modify TPS calculation nor, as a consequence, TPS calculation accuracy.

\section{Conclusions}

We found that TPS dose calculation in the out-of-field dose region depends mostly on the TPS algorithm and TPS model rather than on the commissioned linac. TPSs with CCC calculation algorithm commissioned for different linacs are more likely to return the same dose values than TPSs with different calculation algorithms commissioned for the same linac, especially in highly modulated beams.

Regarding TPS calculation accuracy over the PMK, our results confirm what is reported in AAPM TG 158 and in previous studies [11-13,15,30,31] and show the impact of TPS commissioning: the TPS with the highest calculation accuracy in the out-of-field dose calculation is the one for which the finest tuning in the commissioning process had been performed. Moreover, TPSs' accuracy may depend on beam modulation and on PMK position with respect to the beam edge. These aspects affect the accuracy of DVH dose estimations, too, which are used in clinical practice to quantify the dose received by CIED and to define patient risk class.

Our suggestion is therefore to also perform fine tuning in the out-offield dose region during TPS commissioning to reduce dosimetric uncertainties in the estimation of PMK dose or, if not possible, to estimate these uncertainties with in-vivo/in-phantom measurements. As not all calculation algorithms have the same dependency on beam modulation, a correct estimation of this dependency should be carried out, considering also highly modulated beams. When in-vivo measurements are not feasible, our study shows that measurements on a homogeneous RW3 phantom are suitable as the calculation accuracy in the out-of-field region is not affected by the presence of high-density objects.

\section{References}

[1] Siegel RL, Miller KD, Jemal A. Cancer statistics, 2020. CA A Cancer J Clin 2020;70 (1):7-30. https://doi.org/10.3322/caac.21590.

[2] Marbach JR, Sontag MR, Van Dyk J, Wolbarst AB. Management of radiation oncology patients with implanted cardiac pacemakers: Report of AAPM Task Group No. 34. Med Phys 1994;21(1):85-90. https://doi.org/10.1118/1.597259.

[3] Zecchin M, Morea G, Severgnini M, Sergi E, Baratto Roldan A, Bianco E, Magnani S, De Luca A, Zorzin Fantasia A, Salvatore L, Milan V, Giannini G, Sinagra G. Malfunction of cardiac devices after radiotherapy without direct exposure to ionizing radiation: mechanisms and experimental data. Europace 2016;18(2): 288-93. https://doi.org/10.1093/europace/euv250.

[4] Gauter-Fleckenstein B, Nguyen J, Jahnke L, Wenz F, Gaiser T, Rudic B et al.. Interaction between CIEDs and modern radioteharpy tecniques: Flattening filter free-VMAT, dose-rate effects, scatter radiation, and neutron-generating energies Radiother Oncol. . (in press).

[5] Hurkmans CW, Knegjens JL, Oei BS, Maas AJJ, Uiterwaal GJ, van der Borden AJ, Ploegmakers MMJ, van Erven L. Management of radiation oncology patients with a pacemaker or ICD: A new comprehensive practical guideline in The Netherlands. Radiat Oncol 2012;7(1). https://doi.org/10.1186/1748-717X-7-198.

[6] Prisciandaro JI, Makkar A, Fox CJ, Hayman JA, Horwood L, Pelosi F, Moran JM. Dosimetric review of cardiac implantable electronic device patients receiving radiotherapy. J Appl Clin Med Phys 2015;16(1):254-63. https://doi.org/10.1120/ jacmp.v16i1.5189.

[7] Yan H, Guo F, Zhu D, Stryker S, Trumpore S, Roberts K, Higgins S, Nath R, Chen Z, Liu Wu. On the use of bolus for pacemaker dose measurement and reduction in radiation therapy. J Appl Clin Med Phys 2018;19(1):125-31. https://doi.org/ 10.1002/acm2.12229.

[8] Miften M, Mihailidis D, Kry SF, Reft C, Esquivel C, Farr J, Followill D, Hurkmans C, Liu A, Gayou O, Gossman M, Mahesh M, Popple R, Prisciandaro J, Wilkinson J. Management of radiotherapy patients with implanted cardiac pacemakers and 
defibrillators: A Report of the AAPM TG-203 †. Med Phys 2019;46(12). https://doi. org/10.1002/mp.13838.

[9] Gauter-Fleckenstein B, Israel CW, Dorenkamp M, Dunst J, Roser M, Schimpf R, Steil V, Schäfer J, Höller U, Wenz F. DEGRO/DGK guideline for radiotherapy in patients with cardiac implantable electronic devicesLeitlinie der DEGRO/DGK zur Strahlentherapie bei Patienten mit kardialen implantierten elektronischen Geräten. Strahlenther Onkol 2015;191(5):393-404. https://doi.org/10.1007/s00066-0150817-3.

[10] Zecchin M, Severgnini M, Fiorentino A, Malavasi VL, Menegotti L, Alongi F, Catanzariti D, Jereczek-Fossa BA, Stasi M, Russi E, Boriani G. Management of patients with cardiac implantable electronic devices (CIED) undergoing radiotherapy. Int J Cardiol 2018;255:175-83. https://doi.org/10.1016/j. ijcard.2017.12.061.

[11] Kry SF, Bednarz B, Howell RM, Dauer L, Followill D, Klein E, Paganetti H, Wang B, Wuu C-S, George Xu X. AAPM TG 158: Measurement and calculation of doses outside the treated volume from external-beam radiation therapy. Med Phys 2017; 44(10):e391-429. https://doi.org/10.1002/mp.12462.

[12] Huang JY, Followill DS, Wang XA, Kry SF. Accuracy and sources of error of out-of field dose calculations by a commercial treatment planning system for intensitymodulated radiation therapy treatments. J Appl Clin Med Phys 2013;14(2): 186-97. https://doi.org/10.1120/jacmp.v14i2.4139.

[13] Howell RM, Scarboro SB, Kry SF, Yaldo DZ. Accuracy of out-of-field dose calculations by a commercial treatment planning system. Phys Med Biol 2010;55 (23):6999-7008. https://doi.org/10.1088/0031-9155/55/23/S03.

[14] Wang L, Ding GX. The accuracy of the out-of-field dose calculations using a mode based algorithm in a commercial treatment planning system. Phys Med Biol 2014; 59(13):N113-28. https://doi.org/10.1088/0031-9155/59/13/N113.

[15] Taddei PJ, Jalbout W, Howell RM, Khater N, Geara F, Homann K, Newhauser WD. Analytical model for out-of-field dose in photon craniospinal irradiation. Phys Med Biol 2013;58(21):7463-79. https://doi.org/10.1088/0031-9155/58/21/7463.

[16] Peet SC, Wilks R, Kairn T, Scott B. Crowe, Measuring dose from radiotherapy treatments in the vicinity of cardiac pacemaker. Phys Med 2016;32:1479-87. https://doi.org/10.1016/j.ejmp.2016.11.010.

[17] Severgnini M, Menegotti L, Delana A, Malatesta T, Quattrocchi M, Aslian H, Pietrobon F, Andreoli S, Cattani F, kaiser SR, Eugenia M, Foti C, Falco MD, Guerriero F, Recanello S, Valentini A, Stasi M. [OA177] Contribution of the medical physicist to the italian consensus document on management of patients with cardiac implantable devices (CIED) undergoing radiotherapy and future development from aifm wotking group. Physica Med 2018;52:68. https://doi.org/ 10.1016/j.ejmp.2018.06.249.

[18] Du W, Cho SH, Zhang X, Hoffman KE, Kudchadker RJ. Quantification of beam complexity in intensity-modulated radiation therapy treatment plans: IMRT beam complexity. Med Phys 2014;41(2):021716. https://doi.org/10.1118/1.4861821.

[19] Fippel M. Fast Monte Carlo dose calculation for photon beams based on the VMC electron algorithm. Med Phys 1999;26(8):1466-75. https://doi.org/10.1118/ 1.598676 .
[20] Kry SF, Price M, Followill D, Mourtada F, Salehpour M. The use of LiF (TLD-100) as an out-of-field dosimeter. J Appl Clin Med Phys 2007;8(4):169-75. https://doi. org/10.1120/jacmp.v8i4.2679.

[21] Vlachopoulou V, Malatara G, Delis H, Theodorou K, Kardamakis D, Panayiotakis G. Peripheral dose measurement in high-energy photon radiotherapy with the implementation of MOSFET. World J Radiol 2010;2:434-9. https://doi.org/ 10.4329/wjr.v2.i11.434.

[22] Cheung T, Yu PKN, Butson MJ. Low-dose measurement with a MOSFET in highenergy radiotherapy applications. Radiat Meas 2005;39(1):91-4. https://doi.org/ 10.1016/j.radmeas.2004.04.007.

[23] Scarboro SB, Followill DS, Howell RM, Kry SF. Variations in photon energy spectra of a $6 \mathrm{MV}$ beam and their impact on TLD response: Impact of photon energy spectra on TLD response. Med Phys 2011;38(5):2619-28. https://doi.org/10.1118/ on TLD respon

[24] Kruszyna M, Adamczyk S, Skrobała A, Skórska M, Suchorska W, Zaleska K, Kowalik A, Jackowiak W, Malicki J. Low dose out-of-field radiotherapy, part 1: Measurement of scattered doses. Cancer/Radiothérapie 2017;21(5):345-51. https://doi.org/10.1016/j.canrad.2016.09.017.

[25] Edwards CR, Mountford PJ. Near surface photon energy spectra outside a $6 \mathrm{MV}$ field edge. Phys Med Biol 2004;49(18):N293-301. https://doi.org/10.1088/00319155/49/18/N01.

[26] Van den Heuvel F, Defraene G, Crijns W, Bogaerts R. Out-of-field contributions for IMRT and volumetric modulated arc therapy measured using gafchromic films and compared to calculations using a superposition/convolution based treatment planning system. Radiother Oncol 2012;105(1):127-32. https://doi.org/10.1016/ j.radonc.2011.12.030.

[27] Joosten A, Matzinger O, Jeanneret-Sozzi W, Bochud F, Moeckli R. Evaluation of organ-specific peripheral doses after 2-dimensional, 3-dimensional and hybrid intensity modulated radiation therapy for breast cancer based on Monte Carlo and convolution/superposition algorithms: Implications for secondary cancer risk assessment. Radiother Oncol 2013;106(1):33-41. https://doi.org/10.1016/j. radonc. 2012.11.012.

[28] Sharpe MB. IAEA technical reports series no. 430: commissioning and quality assurance of computerized planning systems for radiation treatment of cancer. Med Phys 2006;33(2). https://doi.org/10.1118/1.2167371. 561-561.

[29] Venselaar J, Welleweerd H, Mijnheer B. Tolerances for the accuracy of photon beam dose calculations of treatment planning systems. Radiother Oncol 2001;60 (2):191-201. https://doi.org/10.1016/S0167-8140(01)00377-2.

[30] Howell RM, Scarboro SB, Taddei PJ, Krishnan S, Kry SF, Newhauser WD. Methodology for determining doses to in-field, out-of-field and partially in-field organs for late effects studies in photon radiotherapy. Phys Med Biol 2010;55(23): 7009-23. https://doi.org/10.1088/0031-9155/55/23/S04.

[31] Yoon J, Heins D, Zhao X, Sanders M, Zhang R. Measurement and modeling of outof-field doses from various advanced post-mastectomy radiotherapy techniques. Phys Med Biol 2017;62:9039-53. https://doi.org/10.1088/1361-6560/aa94b5. 